\title{
[-2]proPSA versus ultrasensitive PSA fluctuations over time in the first year from radical prostatectomy, in an high-risk prostate cancer population: A first report
}

\author{
S. De Luca ${ }^{1}$, R. Passera ${ }^{2 *}$ (D, A. Sottile ${ }^{3}$, C. Fiori ${ }^{1}$, R. M. Scarpa ${ }^{1}$ and F. Porpiglia ${ }^{1}$
}

\begin{abstract}
Background: [-2]proPSA and its derivatives have an higher diagnostic accuracy than PSA in predicting prostate cancer (PCa). In alternative to PSA, ultrasensitive PSA (UPSA) and [-2]proPSA could be potentially useful in recurrent disease detection. This research focused on [-2]proPSA and uPSA fluctuations over time and their possible clinical and pathological determinants, in the first year after RP.

Methods: A cohort of 106 consecutive patients, undergoing RP for high-risk prostate cancer (pT3/pT4 and/or positive margins), was enrolled. No patient received either preoperative/postoperative androgen deprivation therapy or immediate adjuvant RT, this latter for patient choice. [-2]proPSA and UPSA were measured at 1, 3, 6, 9, 12 months after $\mathrm{RP}$; their trends over time were estimated by the mixed-effects linear model. The uPSA relapse was defined either as 3 rising uPSA values after nadir or 2 consecutive UPSA $>0.2 \mathrm{ng} / \mathrm{ml}$ after RP.

Results: The biochemical recurrence (BCR) rate at 1 year after RP was either $38.6 \%$ (in case of 3 rising uPSA values) or $34.9 \%$ (in case of PSA $>0.2 \mathrm{ng} / \mathrm{ml}$ after nadir), respectively. The main risk factors for UPSA fluctuations over time were PSA at diagnosis $>8 \mathrm{ng} / \mathrm{ml}(p=0.014)$, pT $(p=0.038)$ and pN staging $(p=0.001)$. In turn, PSA at diagnosis $>8 \mathrm{ng} / \mathrm{ml}$ $(p=0.012)$ and $\mathrm{pN}(p<0.001)$ were the main determinants for [-2]proPSA trend over time. In a 39 patients subgroup, UPSA decreased from month 1 to 3, while [-2]proPSA increased in $90 \%$ of them; subsequently, both uPSA and $[-2]$ proPSA increased in almost all cases. The [-2]proPSA trend over time was independent from BCR status either in the whole cohort as well in the 39 men subgroup.

Conclusions: Both UPSA and [-2]proPSA had independent significant fluctuations over time. PSA at diagnosis $>8 \mathrm{ng} / \mathrm{ml}$ and pathological staging significantly modified both these trends over time. Since BCR was not confirmed as determinant of [-2]proPSA fluctuations, its use as marker of early biochemical relapse may not be actually recommended, in an high-risk prostate cancer patients population.
\end{abstract}

Keywords: Prostate cancer, (-2)pro-prostate-specific antigen, Prostate-specific antigen, Biochemical recurrence

\section{Background}

After a successful radical prostatectomy (RP), a serum detectable Prostate Specific Antigen (PSA) may be considered a marker of residual prostate tissue, presumably anticipating either locoregional or systemic disease [1].

\footnotetext{
* Correspondence: passera.roberto@gmail.com

${ }^{2}$ Division of Nuclear Medicine, San Giovanni Battista Hospital and University of Torino, Corso AM Dogliotti 14, 10126 Torino, Italy

Full list of author information is available at the end of the article
}

However, approximately $20 \%$ of patients experience biochemical recurrence (BCR) after RP [2-4]; around $30 \%$ of them will ultimately develop a clinical progression $[5,6]$.

Several trials proved that adjuvant radiation therapy (RT) after RP decreases BCR risk and provides survival benefit, in high-risk disease patients. An early and reliable BCR detection is therefore crucial, since postoperative $\mathrm{RT}$ is more effective when given to subjects having low PSA levels [7]. 
New PSA ultrasensitive methods detect levels $<0.1 \mathrm{ng} /$ $\mathrm{ml}$, and some assays even minimal ones $(1 \mathrm{pg} / \mathrm{ml})[8,9]$. A classical definition for ultrasensitive PSA (uPSA) relapse is 3 rising uPSA values after nadir [10]; recurrence seldom occurs in patients with uPSA $<0.04 \mathrm{ng} / \mathrm{ml}$, 3 years after RP [11].

In recent years, many efforts were made to improve the biomarkers diagnostic accuracy for prostate cancer (PCa); at the same time, an alternative to PSA as BCR marker is still unavailable. Some studies showed that [-2]proPSA and its derivatives improve PSA accuracy in predicting $\mathrm{PCa}$ at prostate biopsy $(\mathrm{Bx})$, being associated to $\mathrm{PCa}$ aggressiveness either at $\mathrm{Bx}$ or at final pathology after RP $[12,13]$. Moreover, [-2]proPSA could be potentially useful in recurrent disease detection, a virtually unexplored field.

To our knowledge, no study investigated the $[-2]$ proPSA trend over time post-RP. We enrolled at RP a sequential cohort of high-risk PCa patients (extra prostate disease and/or positive margins), eligible for adjuvant RT but not being given for patient choice.

The primary endpoint of this research was to longitudinally investigate either [-2]proPSA and uPSA time trends as well their clinical and pathological determinants, in the first year after RP, at a single high-volume institution. The secondary endpoint was to elucidate a [-2]proPSA possible role in early BCR detection.

\section{Methods}

A cohort of 106 consecutive patients, undergoing robot assisted radical prostatectomy (RARP) for a pathological high-risk PCa (pT3/pT4 and/or positive margins), was enrolled at San Luigi Gonzaga Hospital - Orbassano (Italy) from September 2013 to October 2014. Among them, 83 patients $(81.3 \%)$ underwent robot-assisted extended pelvic lymph nodes dissection prior to RARP (external iliac artery/vein, obturator fossa, obturator nerve, internal iliac artery/presacral lymph nodes) [14]. Lymphadenectomy was planned according to Briganti nomogram [15]. Pathological staging was performed according to the TNM Classification of Malignant Tumors seventh edition [16]; histological grading was assessed according to the Gleason grading system [17]. No patient received either preoperative/postoperative androgen deprivation therapy or immediate adjuvant RT, this latter for patient choice.

The follow-up was scheduled at 1/3/6/9/12 months after RARP; it included a complete physical and digital rectal examination (DRE), as like [-2]proPSA and UPSA measurements. The uPSA relapse was defined either as 3 rising uPSA values after nadir [10] or two consecutive uPSA $>0.2 \mathrm{ng} / \mathrm{ml}$ rising after RP [18].

Due to the observational nature of this research and according to Italian regulation, no formal IRB/IEC approval was needed [19].
UPSA and [-2]proPSA serum concentrations measurement Serum samples were analysed by the Access 2 Immunoassay System on a UniCell DxI800 instrument (Beckman Coulter, USA). The calibration procedure was performed by a 7-point recombinant [-2]proPSA curve (0-5000 pg/ $\mathrm{ml}$ ). The blank and quantitation limits (according to the Clinical Laboratory Standards Institute document EP17A) were 0.5 and $3.23 \mathrm{pg} / \mathrm{ml}$, respectively. uPSA results were obtained by a single determination, while that from [-2]proPSA by a duplicate one; the analyses were repeated in case of coefficient of variation $>20 \%$. All analyses were performed in the same laboratory (Candiolo Cancer Institute).

\section{Statistical methods}

The primary outcomes were the UPSA and [-2]proPSA trends over time after RARP and their potential modifications by independent covariates. uPSA and [-2]proPSA were longitudinally measured at five time-points $(1 / 3 / 6 / 9$ / 12 months after RP), and these repeated measures were used as dependent variables in univariate and multivariate mixed-effects linear models [20]. Due to the not-Gaussian distribution of uPSA and [-2]proPSA, all models were estimated using their log-transformed values $[\ln (\mathrm{uPSA})$ and $\ln [-2]$ proPSA]. At first, the univariate analyses were performed for the following covariates: age ( $>65$ vs. $\leq 65$ years), Body Mass Index [BMI, (>26 vs. $\leq 26)]$, DRE (positive vs. negative), PSA at diagnosis ( $>8$ vs. $\leq 8 \mathrm{ng} / \mathrm{ml}$ ), number of positive $\mathrm{Bx}$ samples ( $>5$ vs. $\leq 5)$, GS at $\mathrm{Bx}(8-9$ vs. $\leq 7)$, number of lesions at Magnetic Resonance Imaging [MRI ( $\geq 2$ vs. 1 )], prostate volume ( $>40$ vs. $\leq 40 \mathrm{ml}$ ), tumor percentage ( $>10 \%$ vs. $\leq 10 \%)$, GS at surgery (8-9 vs. $\leq 7)$, capsule/vesicles/neural/vascular/marginal involvement (any vs. none), pT (pT3b vs. pT3a vs. pT2), pN (pN+ vs. pN0) and BCR (any vs. none). Two different definitions of BCR were used: either uPSA value $>0.2 \mathrm{ng} / \mathrm{ml}$ or 3 rising uPSA values after nadir. The multivariate mixed-effects linear models for uPSA and [-2]proPSA trends over time were estimated by the restricted maximum likelihood method, using a first-order autoregressive covariance matrix: both $\ln (\mathrm{uPSA})$ and $\ln ([-2]$ proPSA $)$ variances at each time-point were considered comparable and constant, while the correlations between subsequent measures similar. Patient characteristics were analyzed by the Fisher's exact test for categorical variables, while for continuous ones by the Mann-Whitney and Kruskal-Wallis tests (for independent measures) or by the Wilcoxon and Friedman ones (for repeated measures). All results for continuous variables were expressed as the median (range). All reported pvalues were obtained by the two-sided exact method, at the conventional $5 \%$ significance level. Data were analysed as of September 2015 by R 3.2.1 (R Foundation for Statistical Computing, Vienna-A, http://www.Rproject.org). 
Table 1 Main patient characteristics

\begin{tabular}{|c|c|}
\hline Age at diagnosis, years & $65(48-77)$ \\
\hline PSA at diagnosis, $\mathrm{ng} / \mathrm{ml}$ & $7.8(4.0-81.0$ \\
\hline BMl at diagnosis, $\mathrm{kg} / \mathrm{m}^{2}$ & $26.3(17.4-34$ \\
\hline Prostate volume, ml & $41.2(22.4-10$ \\
\hline Tumor percentage, $\%$ & $10.2(2.3-52.9$ \\
\hline \multicolumn{2}{|l|}{ Cancer familiarity, } \\
\hline neg & $6(5.7 \%)$ \\
\hline pos & $100(94.3 \%)$ \\
\hline \multicolumn{2}{|l|}{ DRE, } \\
\hline neg & $71(67.6 \%)$ \\
\hline pos & $34(32.4 \%)$ \\
\hline \multicolumn{2}{|l|}{ GS at biopsy, } \\
\hline 6 & $31(29.5 \%)$ \\
\hline $7(3+4)$ & $29(27.6 \%)$ \\
\hline $7(4+3)$ & $21(20.0 \%)$ \\
\hline 8 & 16(15.2\%) \\
\hline 9 & $8(7.6 \%)$ \\
\hline \multicolumn{2}{|l|}{ Lesions at MRI, } \\
\hline 1 & $31(53.4 \%)$ \\
\hline $2+$ & $27(46.6 \%)$ \\
\hline \multicolumn{2}{|l|}{ GS at surgery, } \\
\hline 6 & $4(3.8 \%)$ \\
\hline $7(3+4)$ & $37(37.4 \%)$ \\
\hline $7(4+3)$ & $37(37.4 \%)$ \\
\hline 8 & 15(15.2\%) \\
\hline 9 & $6(6.1 \%)$ \\
\hline \multicolumn{2}{|l|}{ Margins, } \\
\hline neg & $53(50.0 \%)$ \\
\hline pos & $53(50.0 \%)$ \\
\hline \multicolumn{2}{|l|}{ Capsule involvement, } \\
\hline neg & $25(23.6 \%)$ \\
\hline pos & $81(76.4 \%)$ \\
\hline \multicolumn{2}{|l|}{ Neural involvement, } \\
\hline neg & $8(7.5 \%)$ \\
\hline pos & $98(92.5 \%)$ \\
\hline \multicolumn{2}{|l|}{ Vascular involvement, } \\
\hline neg & $65(68.4 \%)$ \\
\hline pos & $30(31.6 \%)$ \\
\hline pT2 & $9(8.5 \%)$ \\
\hline рТ3а & $83(78.3 \%)$ \\
\hline pT3b & $14(13.2 \%)$ \\
\hline pNo & $69(79.3 \%)$ \\
\hline $\mathrm{pN}+$ & 18 (20.7 \%) \\
\hline
\end{tabular}

Table 1 Main patient characteristics (Continued)

\begin{tabular}{ll}
\hline uPSA, ng/ml & $0.010(0-1.15) / 0.018(0-0.67) /$ \\
$1 / 3 / 6 / 9 / 12$ months & $0.051(0.01-0.52) / 0.100(0.01-0.56) /$ \\
& $0.154(0-0.89)$ \\
[-2]proPSA, pg/ml & $0.22(0-2.14) / 0.57(0-2.84) / 0.89(0-3.97) /$ \\
$1 / 3 / 6 / 9 / 12$ months & $1.25(0.03-3.72) / 1.38(0-4.86)$ \\
\hline
\end{tabular}

For continuous variables, all the results are expressed as median (range)

\section{Results}

The main patient characteristics (106 patients) are reported in Table 1. The 1-year after surgery BCR rate was $34.9 \%$ using uPSA value $>0.2 \mathrm{ng} / \mathrm{ml}$, while $38.6 \%$ using 3 rising uPSA values after nadir; at the same time, no subject had an imaging-confirmed metastatic disease.

The uPSA values sequentially increased in 43.4/69.8/ 83.1/89.0 \% patients, at 3/6/9/12 months after RARP, respectively. The uPSA trend over time was confirmed by the Friedman test $(p<0.001)$; using the Wilcoxon one, all the differences between two adjacent time-points were extremely significant $(p<0.001)$, except that between 1 vs. 3 months $(p=0.833)$.

The mixed-effects linear model was used to confirm the uPSA time trend (within-subject factor) and to estimate its potential risk factors (between-subject factors) (Table 2, Fig. 1a). This approach confirmed that uPSA fluctuations over time were statistically significant $(p<$ 0.001). In the multivariate mixed-effects linear model, the main determinants for uPSA fluctuations were PSA at diagnosis $>8 \mathrm{ng} / \mathrm{ml}(p=0.014), \mathrm{pT}(p=0.038)$ and $\mathrm{pN}$ $(p=0.001)$.

The [-2]proPSA values sequentially increased in $86.8 /$ 93.4/90.4/91.5 \% patients, at 3/6/9/12 months after surgery, respectively. The difference among the median [-2]proPSA repeated measures was highly significant at the Friedman test $(p<0.001)$; all the differences between adjacent time-points were extremely significant $(p<$ 0.001 ), when investigated by the Wilcoxon test.

Using the mixed-effects linear model as for uPSA, [-2]proPSA fluctuations over time were statistically significant $(p<0.001)$ (Table 2, Fig. 1b). Their main predictors were PSA at diagnosis $>8 \mathrm{ng} / \mathrm{ml}(p=0.012)$ and $\mathrm{pN}$ $(p<0.001)$; the interaction between them was marginal $(p=0.099)$.

In 39 patients, uPSA decreased from month 1 to 3 , conversely [-2]proPSA increased in $90 \%$ of them; in the further follow-up, both uPSA and [-2]proPSA increased in almost all cases.

Of note, $[-2]$ proPSA trend over time was independent from BCR status $(p=0.096$ and 0.194 according to BCR definitions, respectively; Fig. 2a, b) in the whole cohort as well in the 39 men subgroup (its BCR rate was $33.3 \%$ ). When BCR was calculated as uPSA $>0.2 \mathrm{ng} / \mathrm{ml}$, the 1 -year [-2]proPSA was around double for $\mathrm{BCR}$ patients 
Table 2 Mixed linear models for UPSA and [-2]proPSA repeated measures

\begin{tabular}{|c|c|c|c|c|c|}
\hline UPSA & Univariate model & Multivariate model & {$[-2]$ proPSA } & Univariate model & Multivariate model \\
\hline Covariate & $p$ & $p$ & Covariate & $p$ & $p$ \\
\hline Time trend & $<0.001$ & $<0.001$ & Time trend & $<0.001$ & $<0.001$ \\
\hline Age $>65$ years & 0.095 & 0.455 & Age $>65$ years & 0.472 & \\
\hline $\mathrm{BMI}>26$ & 0.059 & 0.507 & $\mathrm{BMI}>26$ & 0.315 & \\
\hline PSA at diagnosis $>8 \mathrm{ng} / \mathrm{ml}$ & 0.015 & 0.014 & PSA at diagnosis $>8 \mathrm{ng} / \mathrm{ml}$ & 0.032 & 0.012 \\
\hline Positive DRE & 0.168 & & Positive DRE & 0.241 & \\
\hline Biopsy samples $>5$ & 0.365 & & Biopsy samples $>5$ & 0.483 & \\
\hline GS at biopsy $>7$ & 0.419 & & GS at biopsy $>7$ & 0.349 & \\
\hline MRI lesions $>1$ & 0.178 & & MRI lesions $>1$ & 0.607 & \\
\hline Prostate volume $>40 \mathrm{ml}$ & 0.214 & & Prostate volume $>40 \mathrm{ml}$ & 0.472 & \\
\hline Tumor percentage $>10 \%$ & 0.522 & & Tumor percentage $>10 \%$ & 0.658 & \\
\hline Positive margins & 0.018 & 0.214 & Positive margins & 0.822 & \\
\hline GS at surgery $>7$ & 0.209 & & GS at surgery $>7$ & 0.102 & \\
\hline Capsule involvement & 0.039 & & Capsule involvement & 0.077 & \\
\hline Neural involvement & 0.216 & & Neural involvement & 0.603 & \\
\hline Vascular involvement & 0.110 & & Vascular involvement & 0.083 & 0.952 \\
\hline pT & $<0.001$ & 0.038 & pT & 0.008 & 0.879 \\
\hline \multirow[t]{3}{*}{$\mathrm{pN}$} & $<0.001$ & 0.001 & $\mathrm{pN}$ & 0.008 & $<0.001$ \\
\hline & & & BCR (uPSA +0,2 ng/ml) & 0.096 & \\
\hline & & & $\mathrm{BCR}$ (3 rising uPSA values) & 0.194 & \\
\hline $\mathrm{pT}^{*} \mathrm{pN}$ (interaction) & - & 0.639 & PSA at diagnosis* $\mathrm{pN}$ (interaction) & - & 0.099 \\
\hline
\end{tabular}

$p$-values

compared to no-BCR ones (its observed marginal means were $0.31-0.54-0.87-1.17-1.33 \mathrm{pg} / \mathrm{ml}$ for BCR subjects and $0.41-0.77-1.31-1.64-2.17 \mathrm{pg} / \mathrm{ml}$ for no-BCR ones). When BCR was calculated as 3 rising uPSA values after nadir, [-2]proPSA increased over time with a superimposable pattern $(0.31-0.60-0.93-1.35-1.50 \mathrm{pg} / \mathrm{ml}$ for BCR subjects and $0.37-0.61-1.09-1.32-1.65 \mathrm{pg} / \mathrm{ml}$ for no-BCR ones).

\section{Discussion}

Recently, while many efforts have been made to improve the biomarkers diagnostic accuracy for $\mathrm{PCa}$, an alternative to PSA as BCR marker is still lacking.

A persistently elevated or rising PSA after RP identifies an heterogeneous patient population, having highly variable prognosis and controversial management. Several trials showed that adjuvant RT after RP decreases BCR risk and provides survival benefit for high-risk patients [21-23]. Conversely, surgical treatment would not have failed in up to $50 \%$ of patients receiving adjuvant RT: thus, they would have been unnecessarily exposed to radiations. To minimize overtreatment, RT should be reserved only for confirmed recurrences; however, an early salvage RT has not been proven to be equivalent to adjuvant $\mathrm{RT}$ yet.
uPSA is an interesting tool, whose variations could foresee a BCR [11]; this finding rarely occurs in patients with very low uPSA nadir after RP [24]. However, the uPSA role after RP is not fully defined yet. Recently, Seikkula investigated uPSA after RP and the possible correlation of uPSA doubling time (UPSA-DT) with traditional PSA doubling time (PSA-DT); uPSA $>0.03 \mathrm{ng} / \mathrm{ml}$ emerged as good relapse predictor, while the above correlation between was marginal [25].

Therefore, new biomarkers for risk stratification after RP are required. Several studies demonstrated that the [-2]proPSA truncated form is detectable in tumor extracts and its serum values are markedly associated with $\mathrm{PCa}$ [26-30]. Sokoll demonstrated that [-2]proPSA may improve PCa detection: its raising is associated with an aggressive disease [31]. Le showed that this biomarker was able to discriminate $\mathrm{PCa}$ from benign disease, in males with PSA $2.5-10 \mathrm{ng} / \mathrm{ml}$ and negative DRE [27]. Recently, Guazzoni confirmed that [-2]proPSA and its derivatives are associated with PCa volume and aggressiveness [13].

Nevertheless, well considering that [-2]proPSA and its derivatives have an higher diagnostic performance than PSA, the potential usefulness of this PSA isoform in the detection of recurrent disease post radical treatment is still quite unexplored. 

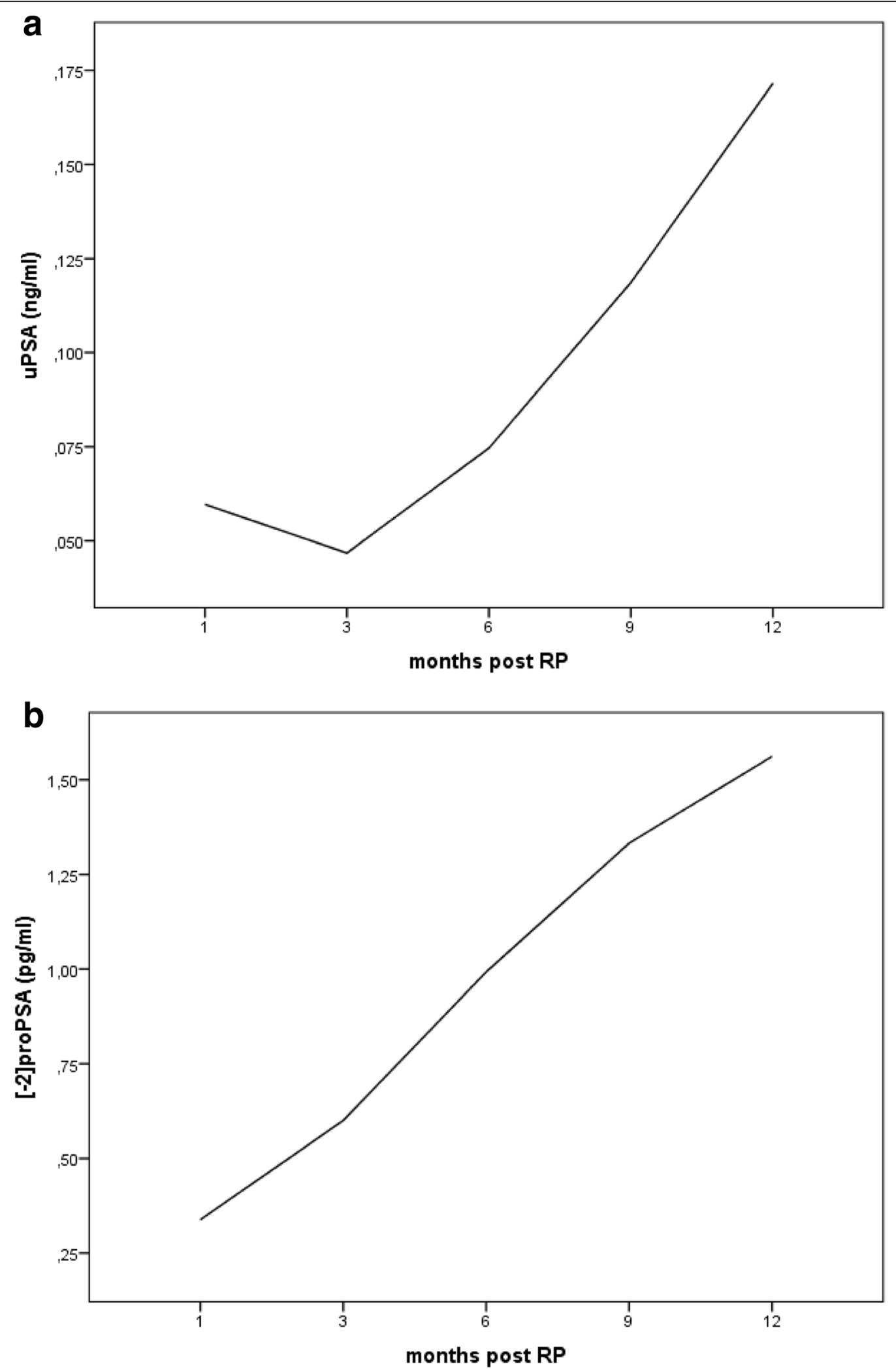

Fig. 1 a, b uPSA and [-2]proPSA mean observed values at 1-3-6-9-12 months after radical prostatectomy 

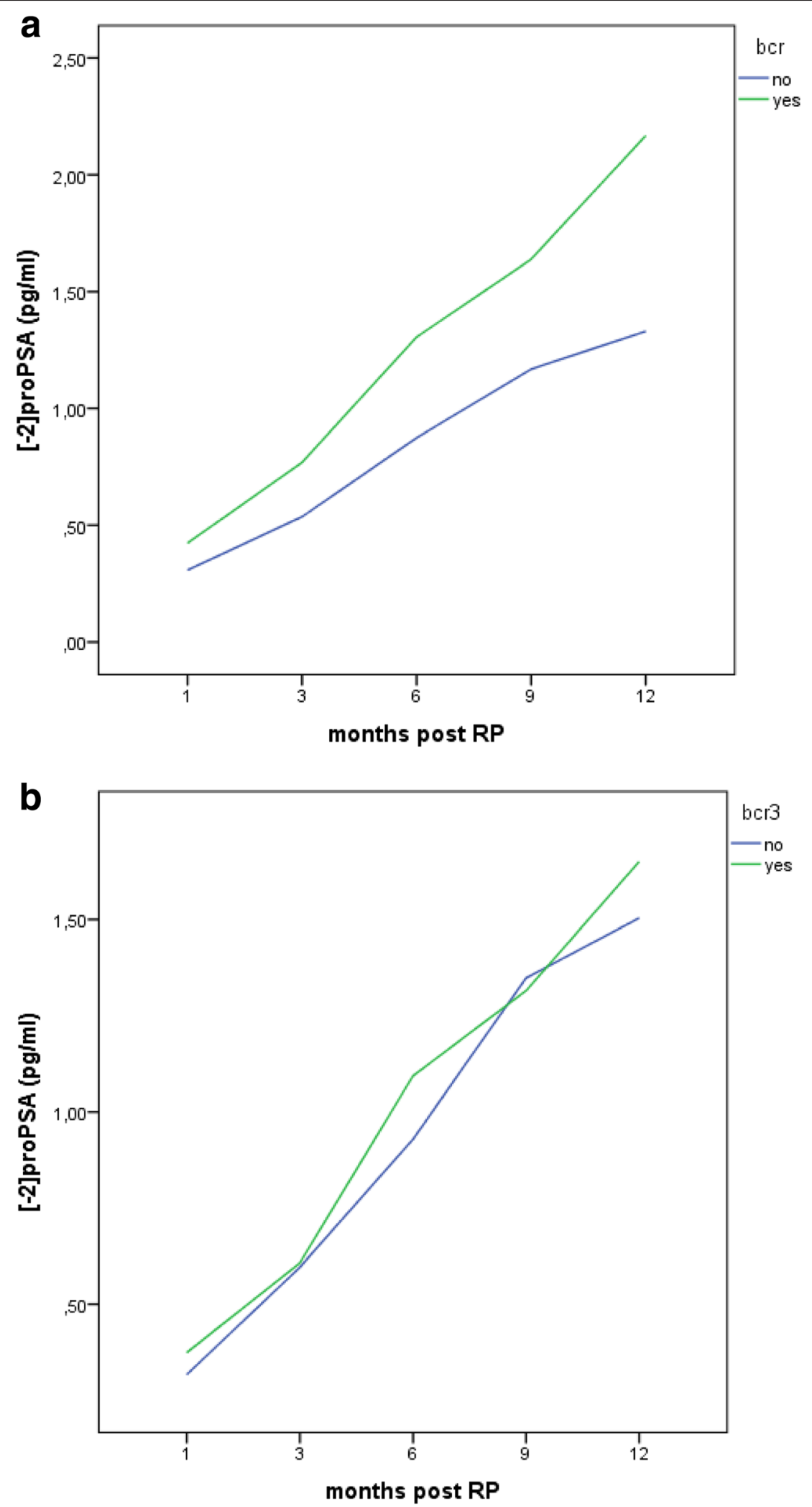

Fig. 2 a, b [-2]proPSA mean observed values at 1-3-6-9-12 months after radical prostatectomy by both BCR definitions (bcr = uPSA value increasing $0.2 \mathrm{ng} / \mathrm{ml} ; \mathrm{bcr} 3=3$ rising uPSA values after nadir) 
Sottile investigated the role of [-2]proPSA in the identification of patients with metastatic progression after RP [32]. In this study, 76 patients with BCR were retrospectively studied; the imaging performed at $\mathrm{BCR}$ time confirmed metastatic disease in 31 out of them. Serum samples were collected at the time of imaging-confirmed metastatic progression. Median PSA, free PSA (fPSA), \%fPSA, [-2]proPSA and PHI were compared between metastatic and non-metastatic patients; [-2]proPSA was a statistically significant predictor of imaging-proven metastatic PCa. However, [-2]proPSA was assessed only at BCR time, so no information may be derived on its potential role, in predicting subsequent clinical progression, when measured at BCR time [32].

The current trial is the first investigating [-2]proPSA fluctuations over time post-RP, and their possible determinants in an high-risk PCa patients cohort.

The [-2]proPSA time trend in the first 3 months showed a different pattern, compared to uPSA one. While uPSA had quite stable levels in two thirds of cases (slowly increasing only in the next period), [-2]proPSA showed a constant linear increase.

The main risk factors for uPSA fluctuations were PSA at diagnosis, $\mathrm{pT}$ and $\mathrm{pN}$ staging, being PSA at diagnosis and $\mathrm{pN}$ the only ones for [-2]proPSA variations.

A secondary endpoint was to investigate [-2]proPSA potential role as BCR early biomarker, in comparison to UPSA: in our series, the [-2]proPSA trend over time was independent from BCR status.

Our study has some points of strengths. The main one is that our results may suggest a stop in further researches for [-2]proPSA in the post RP arena. Second, it was designed as observational study in a homogeneous cohort of men with high-risk PCa, candidates for RP. Finally, we adopted a standardized centralised pathological evaluation; all blood samples were managed in the same laboratory according to Semjonow guidelines: no archived serum were used [33].

\section{Conclusions}

[-2]proPSA and UPSA showed significant fluctuations over time after RP, with an independent pattern. PSA at diagnosis and pathological staging significantly modified both these trends. Since BCR was not confirmed as a modifier of [-2]proPSA time trend, its use as marker of an early biochemical relapse may not be actually recommended, among high-risk prostate cancer patients.

\section{Ethics approval and consent to participate}

Due to the observational nature of this research and according to Italian regulation, the San Luigi Gonzaga Hospital (Orbassano-Italy) IRB/IEC was notified about this research proposal, while no formal ethics approval was needed [19].

\section{Abbreviations}

[-2]proPSA: [-2]pro-prostate specific antigen; BCR: biochemical recurrence: BMI: Body Mass Index; Bx: biopsy; DRE: digital rectal examination; DT: doubling time; GS: Gleason score; LNI: lymph nodes invasion; MRI: magnetic resonance imaging; PCa: prostate cancer; PSA: prostate specific antigen; RARP: robot assisted radical prostatectomy; RP: radical prostatectomy; RT: radiotherapy; uPSA: ultrasensitive prostate specific antigen.

\section{Competing interests}

The authors declare that they have no competing interests.

Authors' contributions

SDL and RP designed the study; SDL and CF collected the clinical data; RP performed the statistical analyses; AS performed the laboratory analyses; RMS and FP supervised the research team. All authors read and approved the final manuscript.

\section{Acknowledgements}

None.

Funding

The authors did not receive any financial support

\section{Author details}

'Division of Urology, San Luigi Gonzaga Hospital and University of Torino, Orbassano, Italy. ${ }^{2}$ Division of Nuclear Medicine, San Giovanni Battista Hospital and University of Torino, Corso AM Dogliotti 14, 10126 Torino, Italy. ${ }^{3}$ Division of Laboratory Medicine, Candiolo Cancer Institute, Candiolo, Italy.

Received: 4 November 2015 Accepted: 16 March 2016

Published online: 24 March 2016

\section{References}

1. Minardi D, Galosi AB, Dell'Atti L, et al. Detectable serum PSA after radical prostatectomy. Clinical and pathological relevance of perianastomotic biopsies. Anticancer Res. 2004;24:1179-85.

2. Polascik TJ, Oesterling JE, Partin AW. Prostate specific antigen: a decade of discovery - what we have learned and where we are going. J Urol. 1999; 162:293-306.

3. Moul JW. Prostate specific antigen only progression of prostate cancer. J Urol. 2000;163:1632-42.

4. Freedland SJ, Humphreys EB, Mangold LA, et al. Risk of prostate cancerspecific mortality following biochemical recurrence after radical prostatectomy. J Am Med Assoc. 2005;294:433-9.

5. Ward JF, Blute ML, Slezak J, et al. The long-term clinical impact of biochemical recurrence of prostate cancer 5 or more years after radical prostatectomy. J Urol. 2003;170:1872-6.

6. Pound $C R$, Partin AW, Eisenberger MA, et al. Natural history of progression after PSA elevation following radical prostatectomy. J Am Med Assoc. 1999; 281:1591-7.

7. Eggener SE, Scardino PT, Walsh PC, et al. Predicting 15 -year prostate cancer specific mortality after radical prostatectomy. J Urol. 2011;185:869-75.

8. Hudson MA, Bahnson RR, Catalona WJ. Clinical use of prostate specific antigen in patients with prostate cancer. J Urol. 1989;142:1011-7.

9. Ferguson RA, Yu H, Kalyvas M, et al. Ultrasensitive detection of prostatespecific antigen by a time-resolved immunofluorometric assay and the immulite immunochemiluminescent third-generation assay: potential applications in prostate and breast cancers. Clin Chem. 1996;42:675-84.

10. Fumitaka S, Tanaka S, Matsuyama Y, et al. Efficiency of ultra-sensitive prostate-specific antigen assay in diagnosing biochemical failure after radical prostatectomy. Jpn J Clin Oncol. 2007;37:446-51.

11. Malik RD, Goldberg JD, Hochman T, et al. Three-year postoperative ultrasensitive prostate-specific antigen following open radical retro-pubic prostatectomy is a predictor for delayed biochemical recurrence. Eur Urol. 2011;60:548-53.

12. Lazzeri $M$, Haese $A$, de la Taille $A$, et al. Serum isoform [-2]proPSA derivatives significantly improve prediction of prostate cancer at initial biopsy in a total PSA range of 2-10 ng/ml: a multicentric European study. Eur Urol. 2013;63(6):986-94. 
13. Guazzoni G, Lazzeri M, Nava L, et al. Preoperative prostate-specific antigen isoform p2PSA and its derivatives, \%p2PSA and prostate health index, predict pathologic outcomes in patients undergoing radical prostatectomy for prostate cancer. Eur Urol. 2012;61(3):455-66.

14. Porpiglia F, Bertolo R, Manfredi M, et al. Total anatomical reconstruction during robot-assisted radical prostatectomy: implications on early recovery of urinary continence. European Urology. 2015. doi: 10.1016/j.eururo.2015.08. 005. [Epub ahead of print]

15. Briganti A, Larcher A, Abdollah F, et al. Updated nomogram predicting lymph node invasion in patients with prostate cancer undergoing extended pelvic lymph node dissection: the essential importance of percentage of positive cores. Eur Urol. 2012;61(3):480-7.

16. Sobin $\mathrm{LH}$, Gospodarowicz MK, Wittekind C. Prostate. In: Sobin LH, Gospodarowicz MK, Wittekind C, editors. UICC TNM classification of malignant tumors. 7th ed. New York: Wiley: 2009. p. 243-8.

17. Epstein Jl, Allsbrook Jr WC, Amin MB, et al. ISUP Grading Committee. The 2005 international society of urological pathology (ISUP) consensus conference on Gleason grading of prostatic carcinoma. Am J Surg Pathol. 2005;29:1228-42

18. Guidelines of prostate cancer. European Association of Urology 2015 (http://uroweb.org/wp-content/uploads/09-Prostate-Cancer_LR.pdf)

19. Agenzia Italiana del Farmaco-AIFA, Guidelines for observational studies, March 202008 (http://www.agenziafarmaco.gov.it/it/content/linee-guidastudi-osservazionali)

20. Singer JD, Willett JB. Applied longitudinal data analysis: modeling change and event occurrence. New York: Oxford University Press; 2003.

21. Stephenson AJ, Shariat SF, Zelefsky MJ, et al. Salvage radiotherapy for recurrent prostate cancer after radical prostatectomy. JAMA. 2004;291(11):1325-32.

22. Stephenson AJ, Scardino PT, Kattan MW, et al. Predicting the outcome of salvage radiation therapy for recurrent prostate cancer after radical prostatectomy. J Clin Oncol. 2007;25(15):2035-41.

23. King CR. The timing of salvage radiotherapy after radical prostatectomy: a systematic review. Int J Radiat Oncol Biol Phys. 2012;84(1):104-11.

24. Kang JJ, Reiter RE, Steinberg ML, et al. Ultrasensitive Prostate Specific Antigen after Prostatectomy Reliably Identifies Patients Requiring Postoperative Radiotherapy. J Urol. 2014. [Epub ahead of print]

25. Seikkula H, Syvänen KT, Kurki S, et al. Role of ultrasensitive prostate-specific antigen in the follow-up of prostate cancer after radical prostatectomy. Urol Oncol. 2014. [Epub ahead of print]

26. Stephan C, Kahrs AM, Cammann H, et al. A [-2]proPSA-based artificial neural network significantly improves differentiation between prostate cancer and benign prostatic diseases. Prostate. 2009;69:198-207.

27. Le BV, Griffin CR, Loeb $S$, et al. [-2]Proenzyme prostate specific antigen is more accurate than total and free prostate specific antigen in differentiating prostate cancer from benign disease in a prospective prostate cancer screening study. J Urol. 2010;183:1355-9.

28. Jansen FH, VanSchaik RHN, Kurstjens J, et al. Prostate-specific antigen (PSA) isoform p2PSA in combination with total PSA and free PSA improves the diagnostic accuracy in prostate cancer detection. Eur Urol. 2010;57:921-7.

29. Guazzoni G, Nava L, Lazzeri M, et al. Prostate-specific antigen (PSA) isoform p2PSA significantly improves the prediction of prostate cancer at initial extended prostate biopsies in patients with total PSA between 2.0 and $10 \mathrm{ng} / \mathrm{ml}$ : results of a prospective study in a clinical setting. Eur Urol. 2011;60:214-22.

30. Catalona WJ, Partin AW, Sanda MG, et al. A multicenter study of [-2]pro-prostate specific antigen combined with prostate specific antigen and free prostate specific antigen for prostate cancer detection in the 2.0 to $10.0 \mathrm{ng} / \mathrm{ml}$ prostate specific antigen range. J Urol. 2011;185:1650-5.

31. Sokoll LJ, Sanda MG, Feng Z, et al. A prospective, multicenter, National Cancer Institute Early Detection Research Network study of [-2]proPSA: improving prostate cancer detection and correlating with cancer aggressiveness. Cancer Epidemiol Biomarkers Prev. 2010;19:1193-200.

32. Sottile A, Ortega C, Berruti A, et al. A pilot study evaluating serum proprostate-specific antigen in patients with rising PSA following radical prostatectomy. Oncol Lett. 2012;3(4):819-24.

33. Semjonow A, Köpke T, Eltze E, et al. Pre-analytical in-vitro stability of [-2]proPSA in blood and serum. Clin Biochem. 2010;47:926-8.

\section{Submit your next manuscript to BioMed Central and we will help you at every step:}

- We accept pre-submission inquiries

- Our selector tool helps you to find the most relevant journal

- We provide round the clock customer support

- Convenient online submission

- Thorough peer review

- Inclusion in PubMed and all major indexing services

- Maximum visibility for your research

Submit your manuscript at www.biomedcentral.com/submit
() BioMed Central 